\title{
Permeation Enhancement of Ketoprofen Using a Supersaturated System with Antinucleant Polymers
}

\author{
Usanee Kumprakob, ${ }^{1)}$ Junichi KaWAKami, ${ }^{*}$ and Isao AdACHI \\ Department of Hospital Pharmacy, Toyama Medical and Pharmaceutical University; 2630 Sugitani, Toyama 930-0194, \\ Japan. Received February 1, 2005; accepted April 28, 2005
}

\begin{abstract}
Permeation enhancement of ketoprofen (KP) from supersaturated systems and the effects of antinucleant polymers on both stability and permeation of supersaturated KP were investigated using silicone membrane as a skin model. The supersaturation was prepared by the cosolvent technique with water and propylene glycol (PG). Saturated solubility of KP in water/PG cosolvent increased markedly with an increase in PG percentage. The time-profiles of the cumulative amount of released KP from supersaturated solutions through the membrane increased linearly, and this KP flux had a significant correlation with the degree of saturation (DS) in $80: 20$, $60: 40,50: 50$, and $40: 60(\mathrm{v} / \mathrm{v})$ water/PG cosolvent systems. The influence of $1 \%$ solutions of antinucleant polymers, hydroxypropylmethylcellulose (HPMC), polyvinylpyrrolidone (PVP), and sodium carboxymethylcellulose (SCMC) on the DS and the stability of supersaturated KP was examined in $60: 40(\mathrm{v} / \mathrm{v})$ water/PG cosolvent. The remaining DS for $24 \mathrm{~h}$ after mixing the solvents increased in the presence of HPMC and SCMC but not PVP. In the presence of SCMC, the physical stability of supersaturated KP was higher, however, the KP flux was lower than that in the control and in the presence of the other polymers. In conclusion, the supersaturation system can be applied to achieve higher transmembrane permeation of KP, and appropriate antinucleant polymers such as HPMC can optimize the physical stability and permeability of KP.
\end{abstract}

Key words supersaturation; antinucleant; ketoprofen; enhancement; polymer; silicone membrane

The efficacy of topically applied drugs is often limited by poor skin penetration. ${ }^{2-4)}$ To improve the cutaneous absorption of drugs, various methods have been developed such as chemical penetration enhancers, novel vehicle systems and physical enhancement techniques including iontophoresis, sonophoresis, and electroporation. ${ }^{3,4)}$ Supersaturated formulations are one of the vehicle systems, and its potential benefit has been examined by many investigators. ${ }^{5,6)}$ Supersaturation is hypothesized to act directly on the drug and to increase skin permeation without structural alternation of the stratum corneum. ${ }^{5,78)}$ The mechanism of permeation enhancement by supersaturation systems is based on increased thermodynamic activity of the drug in the formulation.

A supersaturated state can be prepared by various methods just before or during application. Generally, the following three methods are available to obtain a supersaturated system: (1) by water uptake from the skin, (2) through evaporation of a volatile formulation component during application, and (3) by using the method of mixed cosolvent systems wherein vehicle changes are produced immediately prior to administration of the formulation. ${ }^{3)}$ In the third method, two miscible solvents are chosen, and drugs are stored as a subsaturated solution in the solvent with the higher solubility for the drug. Before use, the other solvent with the lower solubility is added into the subsaturated solution to decrease the overall solubility in the cosolvent mixture. Application of this supersaturated system for hydrocortisone acetate, piroxicam, ibuprofen, oestradiol, and some lipophilic compounds has been investigated ${ }^{2-7)}$ but not for ketoprofen (KP).

As a limiting factor for drug permeation from the supersaturated system, optimization of the degree of saturation (DS) in the basal formulation is very important. For instance, the DS can increase by increasing the drug concentration or by decreasing the overall solubility of drugs in the cosolvent mixture. ${ }^{3)}$ Both approaches can enhance the thermodynamic activity of drugs in the supersaturated systems and thus force drugs out of the vehicle and into the membrane. However, such solutions are thermodynamically unstable and can result in the recrystallization of drugs in the short term. ${ }^{3,7,9,10)}$ As one technique to stabilize the supersaturated systems, the addition of antinucleant polymers such as polyvinylpyrrolidone (PVP), hydroxypropylmethylcellulose (HPMC), sodium carboxymethylcellulose (SCMC), and hydroxypropyl- $\beta$-cyclodextrin has been attempted. ${ }^{7-12)}$ In some conditions, however, these polymers may decrease the release of drugs from the applied formulation into the target membrane due to high stability in the vehicle. ${ }^{7,12)}$ Therefore, the effects of antinucleant polymers on the stability to avoid recrystallization and the permeability of supersaturated drugs should be evaluated.

In the present study, the applicability of the supersaturated system and antinucleant polymers for the permeation enhancement of KP was investigated. A silicone membrane was used as a skin (the stratum corneum) model because its merit in transmembrane study has already been demonstrated by other researchers. ${ }^{3-7,10,12,13)}$ Supersaturated solutions were prepared in cosolvent mixtures of water and propylene glycol (PG). The effects of the antinucleant polymers HPMC, PVP and SCMC on the supersaturated KP were also examined. Finally, the importance of optimization of the physical stability and permeability of KP is discussed.

\section{MATERIALS AND METHODS}

Materials KP was purchased from Wako Pure Chemicals Industries, Ltd. (Japan), silicone membrane of $0.3 \mathrm{~mm}$ thickness was from Shin-Etsu Polymer Co., Ltd. (Japan), and HPMC, SCMC and PVP were obtained from Sigma-Ardlich Japan K.K. (Japan). All other chemicals were purchased from Nacalai Tesque, Inc. (Japan) and of analytical grade.

Solubility in Cosolvent Mixtures A series of water/PG mixtures were prepared from $100 \%$ water to $100 \%$ propylene glycol in $10 \%(\mathrm{v} / \mathrm{v})$ decrements/increments. Saturated solu- 
tions were prepared by adding an excess amount of KP to the mixtures, stirring with a Teflon-coated magnetic bar at $300 \mathrm{rpm}$, and heating in a water bath at $37^{\circ} \mathrm{C}$ for $24 \mathrm{~h}$. The solution was centrifuged to obtain the supernatant for the measurement of saturated KP concentration by HPLC. KP solubility was also examined in water/glycerin cosolvent mixtures.

Physical Stability of the Supersaturated Solutions Supersaturated solutions of KP were prepared using $60: 40$ (v/v) water/PG cosolvent systems, and a $1 \%(\mathrm{w} / \mathrm{v})$ solution of PVP, HPMC, or SCMC was added into the aqueous solvent. Each system was prepared at a DS of 1.02, 2.03, 3.05, 4.07 and 5.08. KP was dissolved in $\mathrm{PG}$ and then mixed with water, 1\% PVP, 1\% HPMC, or 1\% SCMC in test tubes at $37^{\circ} \mathrm{C}$. The changes in the liquid state, such as crystallization and opacity, were observed visually until $24 \mathrm{~h}$. Samples were collected at 2, 4, 6 and $24 \mathrm{~h}$ after mixing the cosolvent. After filtration, the samples were diluted with distilled water and used to analyze the remaining DS of KP.

Permeation from Supersaturated Solutions The release profiles of KP from the supersaturated solutions were studied in a diffusion cell consisting of two $3.0 \mathrm{ml}$ halfcells with a diffusion area of $0.966 \mathrm{~cm}^{2}$ connected with a water jacket to a $37^{\circ} \mathrm{C}$ water bath. ${ }^{15)} \mathrm{A}$ star-head Teflon bar was driven at $300 \mathrm{rpm}$ in each half-cell by a magnetic stirrer. In this permeation experiment, a silicone membrane (0.3 mm thickness) was used as a model of the stratum corneum. $^{3-7,10,12,13)}$ The membrane was cut into an appropriate size $(2 \times 2 \mathrm{~cm})$, soaked in distilled water overnight, and then mounted on a diffusion cell and fastened tightly with a metal clamp. The test solution $(3 \mathrm{ml})$ was introduced to the donor compartment. The receptor compartment was filled with $3.0 \mathrm{ml}$ of distilled water. The amount of drug released was determined by removing $0.5 \mathrm{ml}$ samples at $0.5,1,2,4$, and $6 \mathrm{~h}$. The volume of fluid withdrawn was replaced with fresh distilled water.

Quantitative Determination of KP The concentration of KP was assayed by HPLC. The HPLC system consisted of a pump (LC-6AD, Shimadzu, Kyoto, Japan), ODS-120A TSK-GEL, $4.6-\mathrm{mm} \times 150 \mathrm{~mm}$ column (YMC Co., Ltd., Kyoto), an auto-injector (SIL-9A, Shimadzu), a variable UV detector (SPD-10A, Shimadzu), and an integrator (C-R5A, Shimadzu). The mobile phase consisted of a $0.1 \%(\mathrm{v} / \mathrm{v})$ solution of phosphoric acid and methanol $(50: 50, \mathrm{v} / \mathrm{v})$, and its flow rate was $1.0 \mathrm{ml} / \mathrm{min}$. The column temperature was maintained at $40^{\circ} \mathrm{C}$. Sodium salicylate was used as the internal standard. The detection wavelength was set at $254 \mathrm{~nm}$. The detection limit for KP was $0.1 \mu \mathrm{g} / \mathrm{ml}$.

Statistic Analysis Analysis of variance with Bonferroni's test for multiple comparison was performed, and a statistically significant difference was considered to be $p<0.05$.

\section{RESULTS}

KP Solubility in Cosolvent System Figure 1 shows the saturated solubility of KP in the water/PG (panel A) and water/glycerin (panel B) cosolvent systems at $37^{\circ} \mathrm{C}$. The saturated solubility of KP increased markedly with an increase in the percentage of PG and glycerin. A higher saturated solubility for KP was observed in water/PG than in water/glycerin. The solubility of KP in $100 \%$ PG $(369.1 \mathrm{mg} / \mathrm{ml})$ was al-
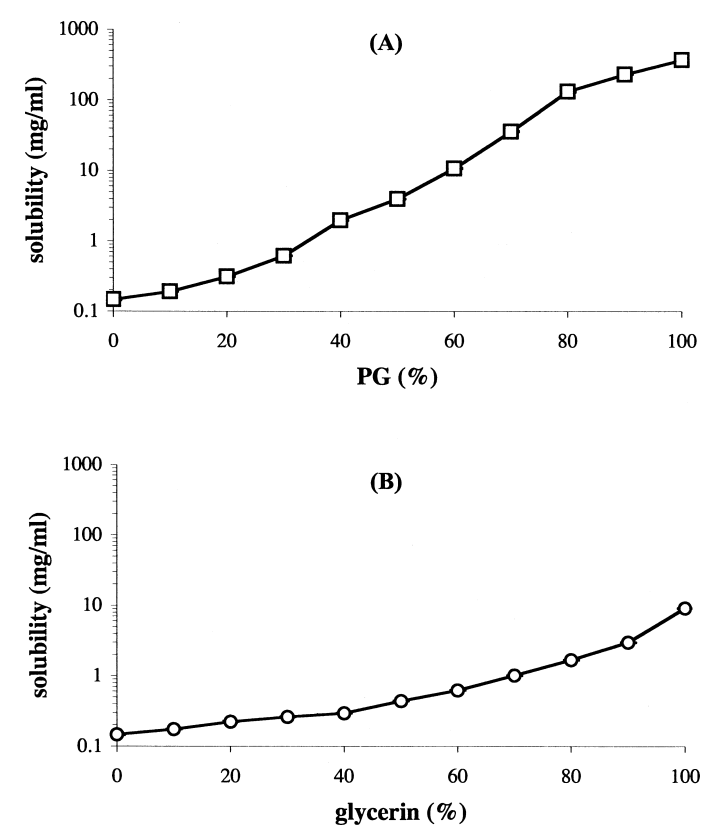

Fig. 1. Solubility of KP in Water/PG (A) and Water/Glycerin (B) Cosolvent System at $37^{\circ} \mathrm{C}$

Each point represents the mean \pm standard deviation (S.D.) of three experiments.

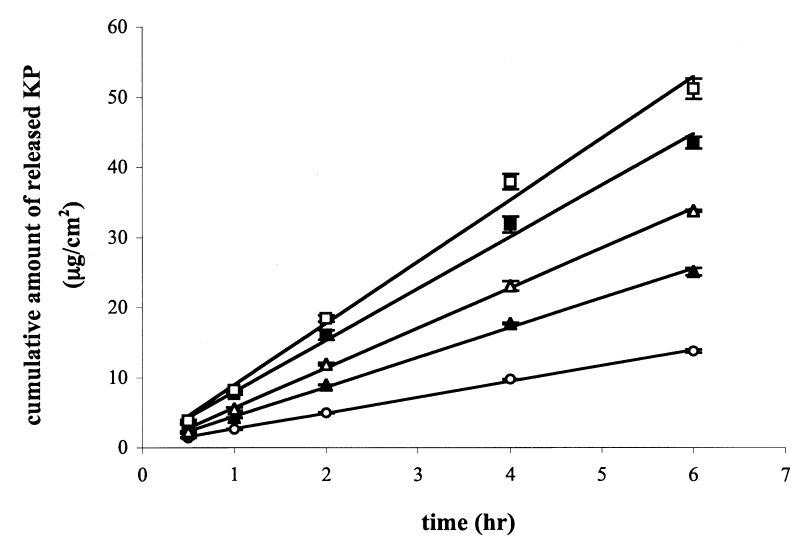

Fig. 2. Time Profiles of Cumulative Release of KP from Supersaturated Solutions in $60: 40(\mathrm{v} / \mathrm{v})$ Water/PG Cosolvent System

Open squares, $\mathrm{DS}=2.03$; closed squares, 1.63 ; open triangles, 1.22 ; closed triangles, 0.81 ; and open circles, 0.41 . The straight lines were obtained by linear regression analysis. Each point represents the mean \pm S.D. of three experiments.

most 2500-fold higher than that in $100 \%$ water.

KP Release from Supersaturated Cosolvent The time profiles of released KP from the supersaturated solution through the membrane were examined. In Fig. 2, the plot between time and the cumulative amount of released KP from supersaturated solutions in the $60: 40(\mathrm{v} / \mathrm{v})$ water/PG cosolvent mixture showed a linear relationship in each DS. The cumulative amount of release of KP increased with an increase in the DS. These linear relationships of the time profiles and the DS-dependent increase in the cumulative amount of released KP from the supersaturated solution were also observed in the $80: 20,50: 50$ and $40: 60(\mathrm{v} / \mathrm{v})$ water/PG cosolvent systems (data not shown).

Relationship between the DS and KP Flux In Fig. 3, the relationship between the DS and the flux of KP from supersaturated solutions was evaluated in various water/PG mixtures. The flux of KP was calculated as the slope of the 
time profile of the cumulative amount of released KP from the supersaturated solutions shown in Fig. 2. KP flux had a linear relationship with the DS in each water/PG cosolvent system. The slopes of the DS-KP flux relations in the $60: 40$ $(\mathrm{v} / \mathrm{v})$ water/PG mixture tended to be higher than that in the other mixtures, however, there were no significant differences among the four groups.

Effects of Polymers on the Stability of Supersaturated KP The physical stability of supersaturated KP in the $60: 40(\mathrm{v} / \mathrm{v})$ water/PG cosolvent system was observed based on visual changes in the liquid state, such as crystallization and opacity (Table 1). In the absence of antinucleant polymers (the control of water/PG cosolvent system), crystallization occurred within $24 \mathrm{~h}$ at a DS of 3.05 or more, and a high amount of KP crystal was observed at the higher DS and the later time. In 1\% PVP/PG and HPMC/PG cosolvent systems, supersaturated KP remained transparent (at $3.05 \mathrm{DS}$ ) or opaque (at 4.07 and 5.08) for $24 \mathrm{~h}$. In the $1 \% \mathrm{SCMC} / \mathrm{PG}$ cosolvent system, the crystallization observed in the control was suppressed, and a lower amount of KP crystal was found at 5.08 DS compared with the control.

To evaluate this stabilization effect of polymers on the

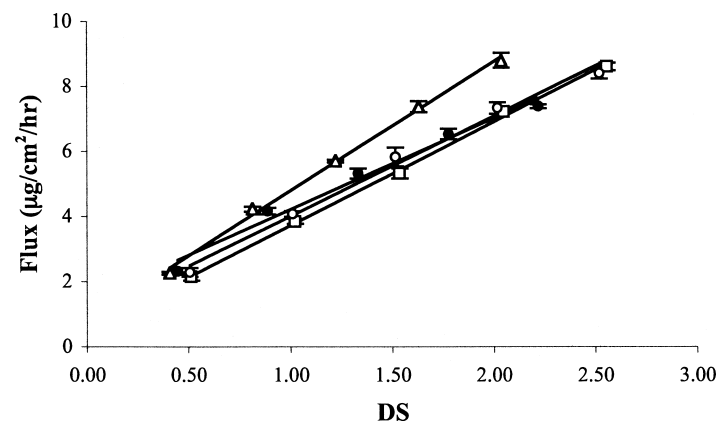

Fig. 3. Relationship between the DS and Flux of KP from Supersaturated Solutions with Various Water/PG Mixtures

Open triangles, water: $\mathrm{PG}=60: 40(\mathrm{v} / \mathrm{v})$; open squares, $80: 20$; open circles, $50: 50$; and closed circles, $40: 60$. The straight lines were obtained by linear regression analysis. Each point represents the mean \pm S.D. of three experiments. physical state of supersaturated KP solution quantitatively, the time profiles of the remaining DS in the cosolvent systems were measured (Fig. 4). The remaining DS decreased drastically at higher DS in the water/PG (panel A) and 1\% $\mathrm{PVP} / \mathrm{PG}$ (panel B) cosolvent systems. In contrast, the remaining DS in 1\% HPMC/PG (panel C) and 1\% SCMC/PG (panel D) cosolvent systems was maintained relatively higher and closer to the initial value than that in the control (panel A).

Effects of Polymers on KP Release from Supersaturated Cosolvent Figure 5 illustrates the time profiles of the cumulative amount of released KP from water/PG cosolvent systems containing 1\% HPMC, SCMC and PVP at 1.22 (panel A) and 2.03 (panel B) DS. A linear increase in the cumulative release of KP was observed in each DS and each antinucleant polymer. HPMC and PVP did not change the KP flux from the control (water). In contrast, SCMC decreased KP flux significantly compared with the other polymers and the control.

\section{DISCUSSION}

Generally, active substances contained in transdermally absorptive formulations can only act after appropriate penetration into and through the cutaneous organ. However, the therapeutic effect of dermatological pharmaceutics is sometimes suboptimal because the transport across the skin is slow due to the barrier function of the stratum corneum, the outermost layer of the skin. ${ }^{15)}$ To overcome this barrier, various methods have been studied such as physical enhancement and penetration enhancement techniques. ${ }^{4)}$ For instance, chemical enhancers and supersaturation were developed to enhance the transport of topically applied drugs across the skin. In the present study, the effects of supersaturated systems and additional antinucleant polymers on the physical stability and transmembrane permeability were investigated using supersaturated $\mathrm{KP}$ as a model compound.

In the solubility study in water/PG and water/glycerin co-

Table 1. Effect of Polymers on Time Profiles of Physical Stability of Supersaturated KP in Water: PG Cosolvent $(60: 40, \mathrm{v} / \mathrm{v})$ at $37^{\circ} \mathrm{C}$

\begin{tabular}{|c|c|c|c|c|c|c|}
\hline Polymers & The DS & $0 \mathrm{~h}$ & $2 \mathrm{~h}$ & $4 \mathrm{~h}$ & $6 \mathrm{~h}$ & $24 \mathrm{~h}$ \\
\hline Control & $\begin{array}{l}1.02 \\
2.03 \\
3.05 \\
4.07 \\
5.08\end{array}$ & $\begin{array}{l}\text { Transparent } \\
\text { Transparent } \\
\text { Transparent } \\
\text { Transparent } \\
\text { Transparent }\end{array}$ & $\begin{array}{l}\text { Transparent } \\
\text { Transparent } \\
\text { Transparent } \\
\text { Crystals + } \\
\text { Crystals ++ }\end{array}$ & $\begin{array}{l}\text { Transparent } \\
\text { Transparent } \\
\text { Transparent } \\
\text { crystals }++ \\
\text { Crystals }+++\end{array}$ & $\begin{array}{l}\text { Transparent } \\
\text { Transparent } \\
\text { Crystals }+ \\
\text { Crystals }+++ \\
\text { Crystals }++++\end{array}$ & $\begin{array}{l}\text { Transparent } \\
\text { Transparent } \\
\text { Crystals }++ \\
\text { Crystals }++++ \\
\text { Crystals }+++++\end{array}$ \\
\hline $1 \%$ PVP & $\begin{array}{l}1.02 \\
2.03 \\
3.05 \\
4.07 \\
5.08\end{array}$ & $\begin{array}{l}\text { Transparent } \\
\text { Transparent } \\
\text { Transparent } \\
\text { Opaque }+ \\
\text { Opaque }++\end{array}$ & $\begin{array}{l}\text { Transparent } \\
\text { Transparent } \\
\text { Transparent } \\
\text { Opaque }+ \\
\text { Opaque }++\end{array}$ & $\begin{array}{l}\text { Transparent } \\
\text { Transparent } \\
\text { Transparent } \\
\text { Opaque }+ \\
\text { Opaque }++\end{array}$ & $\begin{array}{l}\text { Transparent } \\
\text { Transparent } \\
\text { Transparent } \\
\text { Opaque }+ \\
\text { Opaque }++\end{array}$ & $\begin{array}{l}\text { Transparent } \\
\text { Transparent } \\
\text { Transparent } \\
\text { Opaque }+ \\
\text { Opaque }++\end{array}$ \\
\hline $1 \%$ HPMC & $\begin{array}{l}1.02 \\
2.03 \\
3.05 \\
4.07 \\
5.08\end{array}$ & $\begin{array}{l}\text { Transparent } \\
\text { Transparent } \\
\text { Transparent } \\
\text { Opaque }+ \\
\text { Opaque }++\end{array}$ & $\begin{array}{l}\text { Transparent } \\
\text { Transparent } \\
\text { Transparent } \\
\text { Opaque }+ \\
\text { Opaque }++\end{array}$ & $\begin{array}{l}\text { Transparent } \\
\text { Transparent } \\
\text { Transparent } \\
\text { Opaque }+ \\
\text { Opaque }++\end{array}$ & $\begin{array}{l}\text { Transparent } \\
\text { Transparent } \\
\text { Transparent } \\
\text { Opaque }+ \\
\text { Opaque }++\end{array}$ & $\begin{array}{l}\text { Transparent } \\
\text { Transparent } \\
\text { Transparent } \\
\text { Opaque }+ \\
\text { Opaque }++\end{array}$ \\
\hline $1 \% \mathrm{SCMC}$ & $\begin{array}{l}1.02 \\
2.03 \\
3.05 \\
4.07 \\
5.08\end{array}$ & $\begin{array}{l}\text { Transparent } \\
\text { Transparent } \\
\text { Transparent } \\
\text { Transparent } \\
\text { Transparent }\end{array}$ & $\begin{array}{l}\text { Transparent } \\
\text { Transparent } \\
\text { Transparent } \\
\text { Transparent } \\
\text { Transparent }\end{array}$ & $\begin{array}{l}\text { Transparent } \\
\text { Transparent } \\
\text { Transparent } \\
\text { Transparent } \\
\text { Crystals + }\end{array}$ & $\begin{array}{l}\text { Transparent } \\
\text { Transparent } \\
\text { Transparent } \\
\text { Transparent } \\
\text { Crystals + }\end{array}$ & $\begin{array}{l}\text { Transparent } \\
\text { Transparent } \\
\text { Transparent } \\
\text { Transparent } \\
\text { Crystals + + }\end{array}$ \\
\hline
\end{tabular}


(A)

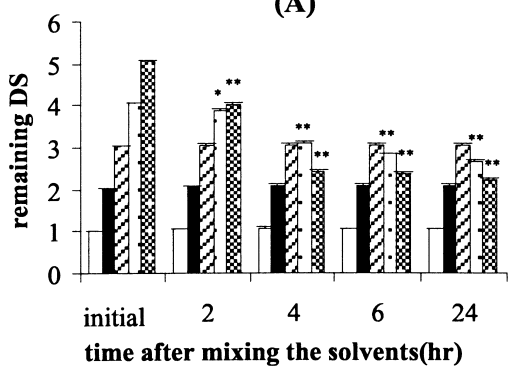

(C)

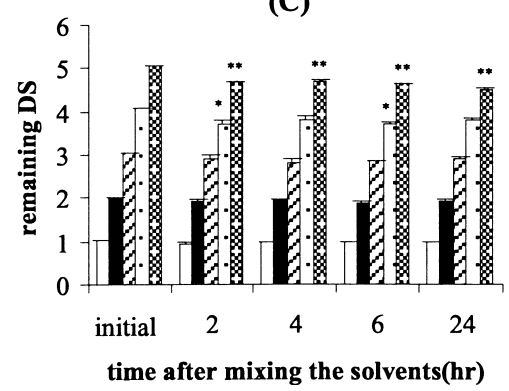

(B)

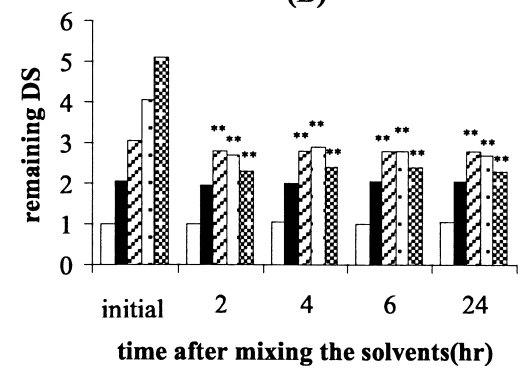

(D)

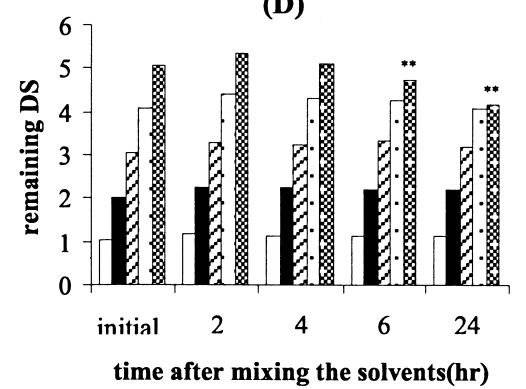

Fig. 4. Time Profiles of Remaining DS after Preparation in $60: 40$ (v/v) Water/PG (A), 1\% PVP/PG (B), 1\% HPMC/PG (C) and 1\% SCMC/PG (D) Open, DS=1.02; closed, 2.03, diagonal, 3.05; light meshed, 4.07; and dark meshed, 5.08. Each column represents the mean \pm S.D. of three experiments.
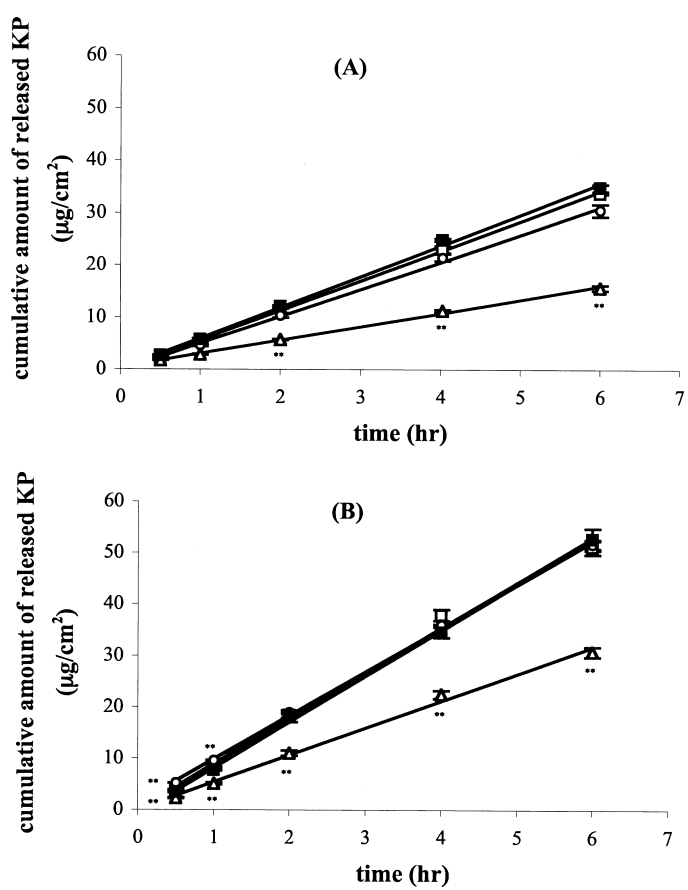

Fig. 5. Effects of Polymers on Time Profiles of Cumulative Release of KP from Supersaturated Solutions at DS of 1.22 (A) and 2.03 (B) in $60: 40(\mathrm{v} / \mathrm{v})$ Water/PG Cosolvent System

Open squares, water/PG cosolvent, closed squares, $1 \%$ PVP/PG; open circles, $1 \%$ $\mathrm{HPMC} / \mathrm{PG}$; and open triangles, $1 \% \mathrm{SCMC} / \mathrm{PG}$. Each point represents the mean \pm S.D. of three experiments.

solvent systems, drastic increases in KP solubility with an increase in the percentage of PG or glycerin and higher solubility in the water/PG than in water/glycerin mixture were observed (Fig. 1). Thus, the water/PG cosolvent system is highly effective at increasing the solubility of KP and was adopted in the following study. This observation was similar with those in previous studies on two other non-steroidal anti-inflammatory drugs, proxicam and ibuprofen. ${ }^{5,7)}$ In the transmembrane permeability study in the water/PG cosolvent system, linear time profiles and a DS-dependent increase in the cumulative amount of released KP were observed (Fig. 2). Moreover, there was no significant difference in the linear relationship between the DS and the KP flux among the various water/PG mixtures (Fig. 3). These findings indicate that $\mathrm{KP}$ flux depends on the DS, at least in the range tested, but not on the actual concentration of applied KP. The saturable concentration (= the concentration at $1 \mathrm{DS}$ ) of KP was different among the $80: 20,60: 40,50: 50$ and $40: 60(\mathrm{v} / \mathrm{v})$ water/ PG cosolvent systems (Fig. 1A). In order to obtain the higher permeation using the supersaturated systems, it is important to increase the DS but not the concentration in applied formulations.

A drawback of the supersaturated systems is that supersaturated drugs tend to crystallize easily due to their thermodynamic instability. To stabilize the supersaturated systems transiently, antinucleant polymers such as methylcellulose can be added to the formulations. In the present study, HPMC, PVP and SCMC were tested as antinucleant polymers. These polymers can adsorb to the hydrophobic surface of compounds, thus increasing the thermodynamic activity and stabilizing the drugs. However, antinucleant polymers may decrease the efficiency of transmembrane drug delivery in some conditions due to their high stability in the vehicle. Therefore, the effects of antinucleant polymers on the stability and release from the supersaturated solution of KP were examined in this study.

Concerning the effects of polymers on the stability of su- 
persaturated KP, the inhibition of recrystallization during $24 \mathrm{~h}$ was visually observed in the presence of HPMC, PVP and SCMC (Table 1). By measuring the time profiles of KP concentration in the cosolvent systems, a higher remaining DS was observed in the presence of HPMC and SCMC but not in PVP (Fig. 4). These findings suggest that HPMC and SCMC can stabilize the physical state of supersaturated KP solution and maintain a higher DS in the short term in water/PG cosolvent systems. Next, the effects of polymers on KP release from the supersaturated systems were examined (Fig. 5). KP release did not change in the presence of HPMC or PVP but decreased in the presence of SCMC. This permeation study in Fig. 5 was performed at relatively lower DS (1.22 and 2.03 in panel A and B, respectively) compared with the previous stability study (Table 1, Fig. 4), therefore, recrystallization and a change in KP concentration did not occur. The reason for the lower permeability in the SCMC/PG cosolvent system may be the higher stability of supersaturated KP that can reduce KP diffusion from the formulation into the membrane. When the antinucleant polymers used in this study were compared, HPMC was found to be better than the other two at optimizing the stability and permeability of supersaturated KP.

In conclusion, a supersaturated system with a water and PG cosolvent mixture was applied to obtain higher permeation of KP. KP flux through the silicone membrane depended on the DS of the supersaturated solution but not the concentration of the KP solution. The effects of antinucleant polymers on the physical stability and release of supersaturated KP were evaluated. HPMC and SCMC increased the stability and remaining DS of supersaturated KP. HPMC did not change the relationship between the DS and KP flux from the supersaturated system, however, SCMC decreased the KP flux. Therefore, optimization of the physical stability and permeability of supersaturated KP is important when selecting appropriate polymers such as HPMC.

\section{REFERENCES AND NOTES}

1) Present address: Department of Pharmaceutical Sciences, Faculty of Pharmacy, Chiangmai University, Chiangmai 50200, Thailand.

2) Moser K., Kriwet K., Froehlich C., Kalia Y. N., Guy R. H., Pharm. Res., 18, 1006-1011 (2001).

3) Moser K., Kriwet K., Froehlich C., Naik A., Kalia Y. N., Guy R. H., J. Pharm. Sci., 90, 607-616 (2001).

4) Moser K., Kriwet K., Naik A., Kalia Y. N., Guy R. H., Eur. J. Pharm. Biopharm., 52, 103-112 (2001).

5) Pellett M. A., Davis A. F., Hadgraft J., Int. J. Pharmaceut., 111, $1-6$ (1994).

6) Davis A. F., Hadgraft J., Int. J. Pharmaceut., 76, 1-8 (1991).

7) Iervolino M., Raghavan S. L., Hadgraft J., Int. J. Pharmaceut., 198, $229-238$ (2000).

8) Iervolino M., Cappello B., Raghavan S. L., Hadgraft J., Int. J. Pharmaceut., 212, 131-141 (2001).

9) Moser K., Kriwet K., Kalia Y. N., Guy R. H., Int. J. Pharmaceut., 224, $169-176$ (2001).

10) Raghavan S. L., Kiepfer B., Davis A. F., Kazarian S. G., Hadgraft J., Int. J. Pharmaceut., 221, 95-105 (2001).

11) Raghavan S. L., Trividic A., Davis A. F., Hadgraft J., Int. J. Pharmaceut., 212, 213-221 (2001).

12) Raghavan S. L., Trividic A., Davis A. F., Hadgraft J., Int. J. Pharmaceut., 193, 231-237 (2000).

13) Yamaguchi Y., Usami T., Natsume H., Aoyagi T., Nagase Y., Sugibayashi K., Morimoto Y., Chem. Pharm. Bull., 45, 537-541 (1997).

14) Geinoz S., Rey S., Boss G., Bunge A. L., Guy R. H., Carrupt P. A. Reist M., Testa B., Pharm. Res., 19, 1622-1629 (2002).

15) Ngawhirunpat T., Hatanaka T., Kawakami J., Adachi I., Biol. Pharm. Bull., 24, 414-417 (2001). 